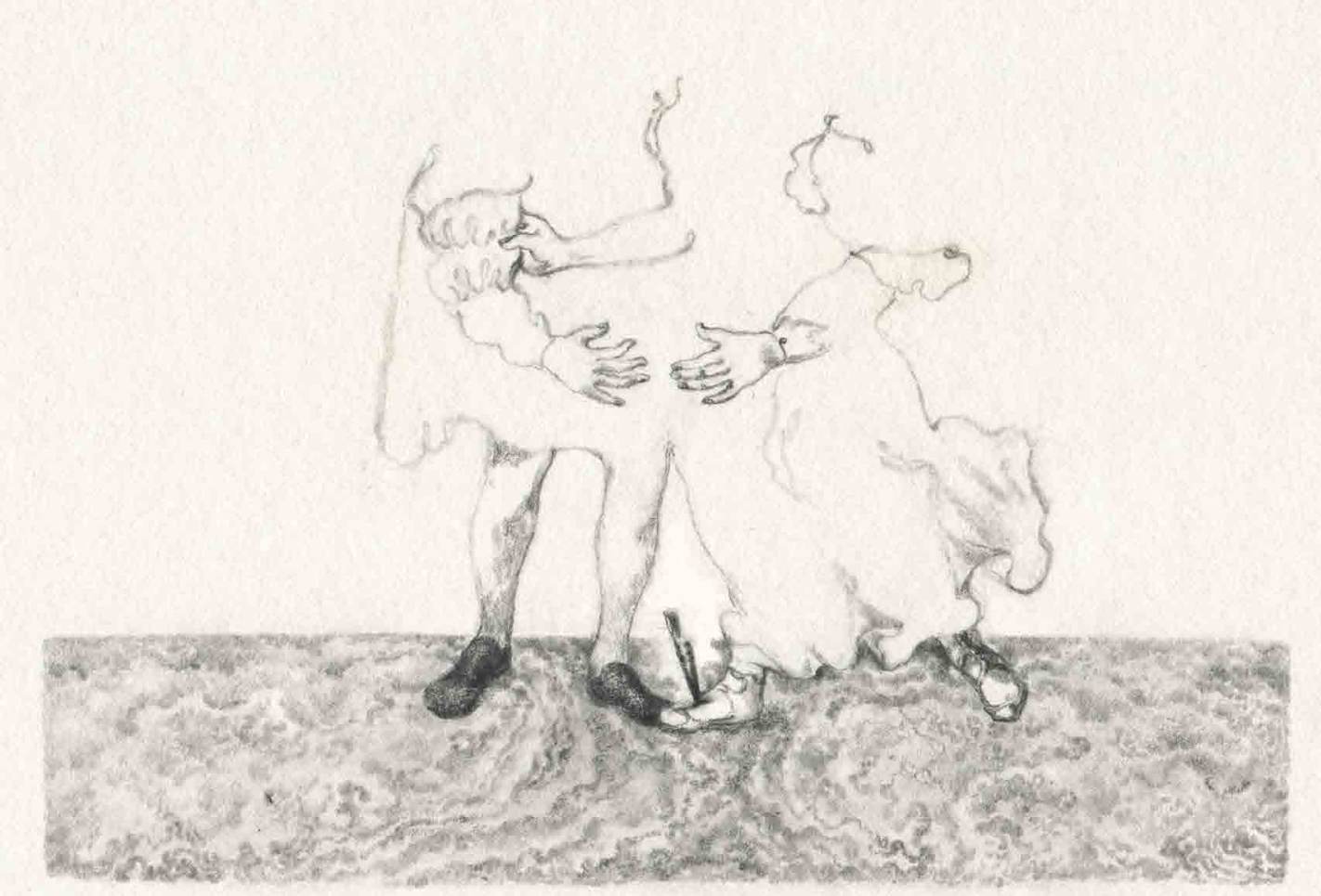

Abbildung: Natalia Kulka

\title{
Kategorienfehler: Antifaschismus im öffentlichen Diskurs und transnationaler Biografik
}

Markus Wegewitz

\begin{abstract}
Zusammenfassung: Dieser Essay nimmt einige Versuche aus der jüngsten Zeit in den Blick, Antifaschismus sowohl als politisches Phänomen als auch als Thema geschichtswissenschaftlicher Forschung zu delegitimieren. An deutschen Universitäten und darüber hinaus bemüht dieser „Anti-Antifaschismus“ die Figur der kommunistischen Kontamination. Um solchen Narrativen entgegenzutreten, schlage ich eine breitere historische Perspektive vor. Diese soll die Pluralität des Antifaschismus, seine verschiedenen Kontinuitätslinien und die Erfahrungen seiner historischen Träger/innen in den Blick nehmen. Plastisch mache ich dieses Argument an der transnationalen Biografie des niederländischen Journalisten Nicolaas Rost.

Schlüsselwörter: Antifaschismus; Biografie; Kommunismus; Historiografie; Transnationale Geschichte
\end{abstract}

\section{Einleitung}

Im Winter 2020 ereignete sich im ostdeutschen Bundesland Thüringen ein Eklat. Nach einer Wahl des Regionalparlaments war absehbar geworden, dass keine der bisher üblichen Koalitionen unter den demokratischen Parteien eine Regierungsmehrheit er- 
reichen würde. Politische Beobachter/innen erwarteten die Einigung auf eine Minderheitsregierung aus Sozialdemokrat/innen, gemäßigten Linken und den Grünen. Stattdessen gelang es der rechtsradikalen „Alternative für Deutschland“ (AfD), die im Landesparlament 22 Prozent der Mandate hielt, die Wahl zum Ministerpräsidenten in ihrem Sinne zu beeinflussen. Zusammen mit den bürgerlich-konservativen Abgeordneten der Christlich Demokratischen Union (CDU) und der neoliberalen Freien Demokratischen Partei (FDP) stimmte die AfD für den Gegenkandidaten zur erwarteten Minderheitsregierung. So kam es, dass der politisch unbedarfte Kleinunternehmer Thomas Kemmerich am 5. Februar 2020 zum Regierungsoberhaupt gewählt wurde. Der FDP-Politiker und Vorsitzende der kleinsten im Parlament vertretenen Partei verdankte die kurzen Tage seiner Amtszeit bis zum Rücktritt am 8. Februar der politischen Finte einer antidemokratischen Partei, an der er sich ohne Not beteiligt hatte. Und ebenso den antikommunistischen Affekten im deutschen Konservatismus. ${ }^{1}$

Die tabuisierte Zusammenarbeit zwischen dem organisierten Rechtsradikalismus mit dem politischen Establishment markierte den Beginn einer Debatte in Politik und Öffentlichkeit, in der vor dem Bruch des „antifaschistischen Konsens“ in der politischen Kultur der Bundesrepublik gewarnt wurde. ${ }^{2}$ Prominent in den sozialen Medien und auch in der analogen Öffentlichkeit bekannte sich etwa die Sozialdemokratische Partei Deutschlands (SPD) zu diesem „Grundkonsens unserer Republik“, pointiert zugespitzt auf den Slogan „Seit über 156 Jahren gilt für die SPD: Kein Fußbreit dem Faschismus!“””

Die Debatte um den normativen Wert des Antifaschismus in Deutschland erreichte mit kurzer Verzögerung auch die Wissenschaft. Neben den etablierten Erklärungsschablonen der politologischen Extremismusforschung (Kusicke, 2020) versuchten sich Historiker/innen beim Ergründen der historischen Dimensionen des Antifaschismus. Die Verbindung zwischen historischer Analyse und politischer Positionierung förderte dabei unterschiedliche Standpunkte zutage, deren kleinster gemeinsamer Nenner auf die Delegitimierung des Antifaschismus-Begriffs hinauslief. Historiker/innen aus dem Umfeld der Historischen Kommission der SPD ${ }^{4}$ kritisierten das Bekenntnis der Partei als „Tendenz zur Beliebigkeit und Simplifizierung“. Ebenso wie der Faschismus solle der Antifaschismus als politische Selbstbeschreibung in der Gegenwart vermieden werden, weil er „nach dem Zweiten Weltkrieg aber durch seine unselige Verknüpfung mit kommunistischen Staatsideologien gewissermaßen seine politische Unschuld verloren" habe (Rother u. a., 2020). An anderer Stelle monierte der Berliner Historiker Jan Behrends den „historisch kontaminierte[n]“ Begriff des Antifaschismus. ${ }^{5}$ Dieser sei ein „Kernbegriff im

1 Zu dieser ideengeschichtlichen Verbindung siehe Großmann (2016, S. 557-559).

2 So argumentierte etwa der Politikwissenschaftler Claus Leggewie (2020).

3 So der Text der Pressemitteilung, die die damaligen Parteivorsitzenden Saskia Esken und Norbert Walter-Borjans (Esken \& Walter-Borjans, 2020) verantwortet haben.

4 Die Kommission wurde 2018 aufgelöst. An ihrer Stelle wurde 2019 das SPD-Geschichtsforum gegründet, dessen Sprecher/innen zwei der Autor/innen des Beitrags sind (Kristina Meyer und Bernd Rother).

5 Das Vokabular der Kontamination verwendete ebenso der Politikwissenschaftler Eckhard Jesse (2021), der sich ein Jahr später immer noch an den Ereignissen in Thüringen abarbeitet. 
globalen Bürgerkrieg der dreißiger Jahre“ und habe schon damals „wenig zur Erklärung der politischen Lage beigetragen." Behrends stellte insbesondere die Nähe zum Stalinismus heraus, die den Begriff „historisch belast[e]“ (Behrends, 2020).

Aversionen gegen den Antifaschismus-Begriff in Deutschland sind keinesfalls neu. Sie gehören seit der „Wiedervereinigung“ zur politischen Rhetorik unterschiedlicher politischer und wissenschaftlicher Milieus in der Berliner Republik und reichen bis zur Gründung der westdeutschen Demokratie zurück (Schildt, 2017). Zudem sind sie Teil eines intellektuellen Trends, der auch in anderen europäischen Ländern wie Frankreich oder Italien Fuß gefasst hat. ${ }^{6}$ Die behauptete Übereinstimmung zwischen Kommunismus, Stalinismus und Antifaschismus ist eine lang bemühte Binsenweisheit, die aktuell einmal mehr Konjunktur hat. Man mag zurückdenken an die Welle von akademischen Abwicklungsbemühungen am Beginn der 1990er Jahre, die die Demontage der Staatsdoktrin der DDR auf den Begriff des Antifaschismus per se ausgedehnt hatten. Am deutlichsten trat dieser Impetus damals vielleicht in der Studie der Politikwissenschaftlerin Antonia Grunenberg zu Tage, die mit Antifaschismus. Ein deutscher Mythos eine intellektuelle Blaupause für die Mär der Wesensverwandtschaft des Faschismus mit seinen erklärten Gegner/innen vorgelegt hatte (Grunenberg, 1993, S. 204-209). Enzo Traverso, der die französische und italienische Historiografie und Intellektuellengeschichte nach ähnlichen Paradigmen durchforstet hat, beschreibt dieses Phänomen als „Anti-Antifaschismus“. Historiker/innen, die nach diesem Paradigma arbeiten, nehmen laut Traverso für sich eine scheinbare Objektivität und Unparteilichkeit in Anspruch, die sie ihren vermeintlichen Gegner/innen absprechen. Ferner klassifizierten sie Antifaschismus als Spielart des Totalitarismus, argumentierten mit der Gleichsetzung von faschistischer und antifaschistischer Gewaltpraxis und verabsolutierten eine Position der politischen Äquidistanz (Traverso, 2016a, S. 328).

Die Pluralität des Antifaschismus bei seiner gleichzeitigen, aber nicht ausschließlichen Strukturierung durch linke Parteien und Bewegungen kann mit einem solchen verengten Blick auf die Vergangenheit weder erkundet noch verstanden werden. So steht den intellektuellen Kurzschlüssen des Anti-Antifaschismus denn auch ein eklatanter Mangel an empirischer Forschung gegenüber. ${ }^{7}$ Angesichts der historischen Bedeutung des Antifaschismus als Bündel an sozialen Bewegungen, als politisches Mobilisierungsmoment und Ausgangspunkt von erinnerungspolitischen Narrativen ist dieser Befund erstaunlich. In seiner Vernetzung zwischen politischen Parteien, Bewegungen, Massenorganisationen und intellektuellen Netzwerken ist der Antifaschismus zudem kaum als uniformes Phänomen zu fassen. Bestimmungen des Begriffs sind und waren oft nur Definitionen mittlerer Reichweite (Wegewitz, 2021, S. 14). Gegen eine undiffe-

6 Zu nennen ist hier etwa für Italien das einflussreiche Werk François Furets (Furet, 1995) und der Revisionismus des Mussolini-Biografen Renzo di Felice in Italien (De Felice, 1995).

7 Späth (2019) bietet einen guten Überblick zum internationalen Forschungsstand. Neuere akademische Studien zum Antifaschismus entstanden in den letzten Jahren aber größtenteils außerhalb des deutschsprachigen Wissenschaftsbetriebs, siehe etwa Braskén, Copsey und Featherstone (Braskén u. a., 2020). 
renzierte Abqualifizierung des Antifaschismus spricht ebenfalls die globale Reichweite des Phänomens, die in vielen verschiedenen lokalen und nationalen Kontexten ganz unterschiedliche politische Bündnisse und Partizipationsformen hervorgebracht hat (Fitch u. a., 2020, S. 2). Ebenso hält die Erforschung des Antifaschismus Erkenntnisse über zentrale Identitätskonstruktionen und Gesellschaftskonzepte im politischen 20. Jahrhundert bereit (Bray u. a., 2020, S. 6).

Um die Pluralität und Relevanz des Antifaschismus im langen 20. Jahrhundert zu dokumentieren und gegen eine präsentistische Ignoranz bei der Erforschung des Phänomens anzuschreiben, möchte ich in diesem Text allerdings keine Ideengeschichte versuchen. Stattdessen stelle ich Schlaglichter aus einer antifaschistischen Biografie vor. Sie zeigen eine Erfahrungsgeschichte des kommunistischen Antifaschismus auf, die sich zwischen den Jahren vor dem Beginn der nationalsozialistischen Herrschaft in Deutschland und dem Tod Stalins 1953 erstreckt. Protagonist meiner Ausführungen ist der niederländische Journalist Nicolaas Rost, dessen Wirken ich in meiner Dissertation erschlossen habe. ${ }^{8}$ Insbesondere geht es dabei um die Bestimmung des Verhältnisses zwischen dieser Variante des Antifaschismus und den kommunistischen Parteien in Europa, die in diesem Zeitraum mehrheitlich zu stalinistischen Kaderorganisationen umgebaut wurden. Ebenso wichtig ist der Fokus auf der aktivistischen Kontinuität über verschiedene Organisationsformen und Zäsuren hinweg, der die forschungsökonomische Trennung zwischen dem "historischen“ Antifaschismus vor 1945 und seinem Nachleben nach der Befreiung Europas negiert. ${ }^{9}$

\section{Antifaschistische Lebenswelten}

Nicolaas Rost war 31 Jahre alt, als er in die Kommunistische Partei der Niederlande eintrat (Rost, 1951). Diese Entscheidung war für inn die Konsequenz seiner Karriere als politischer Reporter im Deutschland der Zwischenkriegszeit. Begonnen hatte seine Politisierung in den letzten Tagen des Spartakus-Aufstandes, die er als junger Journalist in Berlin wahrgenommen hatte (Rost, 1919). Seit diesen Tagen bewegte er sich in den linken politischen Milieus der Weimarer Republik und knüpfe darüber hinaus Kontakte in den transnationalen Bewegungen und Massenorganisationen der Zwischenkriegszeit. Hier mischten sich Hoffnungen auf die Revolution, wie sie das Beispiel der Sowjetunion verkörperte, mit neuen Formen der politischen Vergemeinschaftung. Organisationen wie die Internationale Arbeiterhilfe (IAH) prägten in dieser Zeit einen inklusiven Begriff

8 Nicolaas Rost, geboren 1896 in Groningen, gestorben 1967 in Amsterdam, lebte lange Zeit in Berlin, war Kulturjournalist, Kommunist und Widerstandskämpfer. Er war wahrscheinlich der erste Niederländer, der 1933 in einem deutschen Konzentrationslager gefangen gehalten wurde und überlebte das KZ Dachau. Sein Leben beschriebt Hans Olink (1997) exzellent auf Niederländisch. Zu Rosts transnationaler Biografie siehe auch meine eigene Studie (Wegewitz, 2021, S. 20-25).

9 Zur fehlenden Betrachtung der Kontinuitäten im Antifaschismus siehe Bray (2017, S. xviii). 
der politischen Solidarität und verbanden inn mit neuen Formen des politischen Aktivismus und einer modernen Kommunikationspraxis (Braskén, 2015, S. 23-28). In der jungen Sowjetunion waren es Organisationen wie die Internationale Rote Hilfe, die eine übernationale Solidargemeinschaft propagierten und umsetzten (Albert, 2017, S. 347). Als Unterstützungsorganisationen der kommunistischen Parteien, aber auch als transnationale Handlungs- und Kommunikationsräume schlossen solche Strukturen eine Vielzahl von kleineren Gruppen und intellektuellen Zirkeln mit ein. Sie waren der soziale Ort einer tiefgreifenden Politisierung, die sich sowohl innerhalb als auch außerhalb der Disziplin des Parteikommunismus vollzog. Innerhalb dieses Raumes verbanden Exponenten wie Rost die Niederlande mit Diskursen in nahezu allen europäischen Gesellschaften. Sie knüpften Verbindungen, die in den 1930er Jahren wichtig werden sollten, als die Niederlande zum Rückzugsort vor der nationalsozialistischen Gewalt wurden und sich die organisatorische Infrastruktur des Antifaschismus in die Exilländer verlagerte. In Rosts Selbstverständnis hatte inn dieses Engagement vom Land seiner Geburt entfremdet. „Denn wer Holland liebt muss es gleicherzeit [sic!] unendlich hassen“, schrieb er einmal (Rost, 1926, S. 584). In der Inszenierung seiner politischen Biografie stellte er die Distanz zu nationalen Zugehörigkeiten heraus.

In den ausgehenden 1920er Jahren war der Widerstand gegen die Bewegung Mussolinis in Italien und gegen den Nationalsozialismus im Deutschen Reich in diesen Milieus zunächst ein Thema unter vielen. Antifaschismus, wie die Gegnerschaft ab 1923 in Organisationen wie dem Internationalen Aktionskomitee gegen Krieg und Faschismus benannt wurde (Braskén, 2016, S. 580), stand neben Aktionsfeldern wie der internationalen Frauenbewegungen, der Gewerkschaftsinternationale, pazifistischen und antikolonialen Engagement (Bayerlein u. a., 2017, S. 8-10; Petersson, 2013, S. 187-189). Abhängig von der politischen Opportunität wurde dieser Impetus der emanzipatorischen Bewegungen auch von der Kommunistischen Internationale (Komintern) aufgegriffen, deren Spitzenfunktionäre wie Willi Münzenberg in diesem Feld die Grundlagen einer Bündnispolitik vorbereiteten, die in den 1930er Jahren wichtig werden sollten (Featherstone, 2018, S. 48-49). Dieser von Anfang an transnationale politische Raum ist bisher ungenügend von der historischen Forschung betrachtet worden. Das mag auch daran liegen, dass er mit Perspektiven, die nach einer parteikommunistischen Dominanz oder Kontaminierung suchen, kaum wahrgenommen werden kann.

Der Weg von Nicolaas Rost in diese antifaschistischen Lebenswelten führte über die deutsche Schriftkultur. In Berlin war er über seine Berichterstattung mit linken Sympathien an die expressionistische Zeitschrift „Die Aktion“ geraten. ${ }^{10}$ Dort eignete er sich den Anspruch an, Kultur als Werkzeug zur politischen Diagnose und Veränderung der gesellschaftlichen Wirklichkeit zu nutzen. Verstärkt wurde dieses Selbstbild durch die

10 „Die Aktion“ erschien in ihrer damaligen Form von 1912 bis 1932. Unter der Redaktion von Franz Pfemfert verband die Zeitschrift in Deutschland die Kritik an der Neuen Sachlichkeit mit einem undogmatischen Linksradikalismus. Insbesondere ihre Positionierung gegen die Sozialdemokratie und die Aufrüstung beeinflusste zahlreiche Intellektuelle in der Weimarer Republik (Raunig, 2017, S. 184-191). 
Mitarbeit im Arbeiterschreiber-Kollektiv „links richten“, das ein breites Spektrum von Journalist/innen, Schriftsteller/innen und Künstler/innen vereinte. Dem Kollektiv gehörten unter anderem die Autorin Henriëtte Roland Holst ${ }^{11}$ und der Philosoph Gerrit Mannoury ${ }^{12}$ an. Viele seiner Mitglieder pflegten ein Selbstverständnis als kritische Avantgarde und verorteten sich in globalen politischen Konflikten wie den Auswirkungen der Oktoberrevolution und dem Kampf um die Unabhängigkeit in den niederländischen Kolonialgebieten. Verbreitert wurde Rosts antifaschistischer Horizont auch durch Zeitschriftenprojekte wie die „Weltbühne“, die - ähnlich wie der Zirkel um den italienischen Philosophen Benedetto Croce und der linksrevolutionäre Surrealismus in Frankreich zu den ersten antifaschistischen Sammlungsbewegungen in der Zwischenkriegszeit gehört, die explizit nicht kommunistisch orientiert waren (Traverso, 2016b, S. 261-262).

\section{Ambivalenzen der Volksfront}

Begreift man Antifaschismus nicht als einen eindeutig definierbaren Begriff (Copsey, 2010, S. xviii), sondern als Mobilisierungsvokabel, die unterschiedliche historische Akteur/innen, politische Organisationen und Lebenswelten miteinander verbindet, wird die Vielschichtigkeit dieses Phänomens deutlich. Ausgehend von der Bestimmung des Faschismus als seinem politischen Gegenüber, war die Pluralität des Antifaschismus in der Regel so breit wie seine politische und soziale Basis. ${ }^{13}$ Eine der wichtigsten Strukturierungen dieser Pluralität in den 1930er Jahren war die Idee der Sammlung aller Gegner/innen von Faschismus und Nationalsozialismus in einer gemeinsamen Organisationsstruktur. Die Idee eines breiten, die verschiedenen Parteien und Lager übergreifenden Bündnisses wurde in den 1930er Jahren mit dem Konzept der Volksfront aufgegriffen, das die kommunistischen Parteien als offizielle Linie erst nach dem Beginn des nationalsozialistischen Regimes in Deutschland annahmen. Die Idee der Volksfront zirkulierte in den 1920er Jahren in verschiedenen kommunistischen Diskursen, die mit einer taktischen Zusammenarbeit auch außerhalb der eigenen Partei auf die wenig revolutionären Zustände in den europäischen Staaten zu reagieren versuchten. Ein solcher legitimatorischer Bezug auf das Volk war insbesondere in der deutschen Politik am Ende der Weimarer Republik allgegenwärtig (Langkau-Alex, 2004, S. 4-5) und konkurrierte mit Konzepten wie der „Volksgemeinschaft“, die neben der politischen Einheit

11 Geboren 1869 in Noordwijk, gestorben 1952 in Amsterdam. Roland Holst war Anfang des Jahrhunderts eine der wichtigsten sozialistischen Schriftstellerinnen in den Niederlanden und übersetzte unter anderem die Internationale ins Niederländische.

12 Geboren 1867 in Wormerveer, gestorben 1956 in Amsterdam. Er war Mathematiker und kommunistischer Intellektueller. In den Niederlanden gehörte Mannoury zu den führenden Kritikern der Stalinisierung in der Sowjetunion.

13 Diesen Punkt macht García (2016, S. 97) für die Kultur des Antifaschismus im Spanischen Bürgerkrieg. Meine These ist, dass dieser Befund auch darüber hinaus gilt.

SLH 10/2021 | p. 6 of 18 
zugleich seine Homogenität versprachen (Wildt, 2019, S. 60). Im Gegensatz dazu war die Volksfront ein Zweckbündnis, das eine vorpolitische Homogenität des Volkes nicht deklarierte.

Schon in den Jahren vor ihrer offiziellen Adaption hatten Antifaschist/innen eine solche Bündnispolitik praktiziert (Bayerlein, 1996, S. 111-112). Für Rost, der in der Welle der politischen Gewalt nach dem Reichstagsbrand in der Nacht vom 27. zum 28. März 1933 in das Konzentrationslager Oranienburg verschleppt worden war, war diese Bündnispolitik schon zu diesem Zeitpunkt Kern seiner Aktivitäten. In seinen politischen Berichten über seine Gefangenschaft schrieb er von der Einheit zwischen Kommunisten, Sozialdemokraten, Trotzkisten, Gewerkschaftern und jüdischen Verfolgten, die sich aus der gemeinsam durchlittenen Erfahrung ergeben müsse: „Was bis jetzt weder einer Partei noch der revolutionären Presse gelang, wurde unter den Schlägen der SA Wirklichkeit [...]", befand er (Rost, 1933, S. 16).

In der historischen Entwicklung des Antifaschismus entfaltete dieses Bündnis solcher Propaganda zum Trotz allerdings nie die Wirksamkeit, die inm offiziös etwa von der Komintern zugeschrieben wurde. Unter der kommunistischen Ägide, während der Stalinisierung, den Schauprozessen und der pragmatischen Machtpolitik der Sowjetunion herrschte immer eine Gleichzeitigkeit von Miteinander und Abgrenzung, die jegliche Volksfront zu einem prekären Projekt werden ließ (Hobsbawm, 1985, S. 247). Die beschworene Handlungseinheit ließ sich, etwa in den ersten Jahren des Spanischen Bürgerkrieges oder während der Front populaire in Frankreich, nur anlassbezogen und für kurze Dauer erreichen. Der Moment des Scheiterns war ein ständiger Begleiter der antifaschistischen Organisationsbemühungen - vom beinahe widerstandslosen Regierungsantritt Hitlers über die verlorene Abstimmung zur Angliederung des Saarlands an das nationalsozialistische Deutschland 1935 bis zur Niederlage in Spanien 1939.

Dennoch war das Hinarbeiten auf die Vereinigung der Kern des individuellen und kollektiven Engagements vieler Antifaschist/innen. Aus diesem Gedanken gingen zahlreiche neugegründete Organisationen hervor, die ein Angebot zur Mitarbeit an alle politischen Lager machten. Rost und andere begründeten mit ihren Aktivitäten so ein weitgespanntes, integratives Netzwerk. Organisationsbemühungen standen zumeist unter einem kommunistischen Führungsanspruch. Antifaschist/innen propagierten ebenfalls die Zusammenarbeit mit liberalen, konservativen und christlichen Gruppen, die sich in einigen Fällen - zumeist anlassbezogen und vor dem Hintergrund einer Bedrohung der nationalen Souveränität - umsetzen ließ (Barrett, 1999; Karimäki, 2019, S. 46). Auch Organisationen wie das Comité de Vigilance des Intellectuels Antifascistes in Westeuropa oder die IAH waren in den 1930er Jahren in der Lage, dutzende Intellektuelle und Exilierte in Frankreich, Großbritannien, Polen oder der Tschechoslowakei in ihren Kampagnen zur Mitarbeit zu bewegen (Braskén, 2015, S. 2; Racine-Furlaud, 1977, S. 99).

Die Verbreiterung der politischen Basis für eine Volksfront war auch auf der individuellen Ebene sinnstiftend für viele Antifaschist/innen. Die in dieser transnationalen 
Gemeinschaft hochgehaltenen Weltaneignungsformeln wie Humanität, Demokratie und Pazifismus entfalteten enormes Mobilisierungspotential, auch und gerade bei den Menschen, die in den europäischen Diktaturen verfolgt und vertrieben worden waren (Koch, 2020, S. 224-225). Temporäre Erfolge, unter diesen Maximen ein handlungsfähiges politisches Kollektiv zu versammeln, hingen auch mit der Nutzbarmachung des Kulturlebens zusammen. Künstler/innen und Kunstwerke und insbesondere die Schriftkultur liehen dem Antifaschismus in den Nachbarstaaten des nationalsozialistischen Deutschlands ihre Legitimität. Der Zusammenhalt der diffus organisierten, aber resilienten politischen Kultur des Antifaschismus gründete auf dem manichäischen Gestus der Verteidigung der Kultur gegen den als Barbarei essentialisierten Faschismus. Rabinbach beschreibt diese Unterscheidung als grundlegend für jedes politische Urteil, das in der Perspektive der Antifaschist/innen die Welt in zwei verfeindete Lager teilte (Rabinbach, 2009, S. 35). Ambivalenzen in der Interpretation - etwa der Gedanke, den Nationalsozialismus als ein modern organisiertes, gleichwohl verbrecherisches Regime zu begreifen, das ebenfalls mit kulturpolitischen Legitimationsstrategien arbeitete - gingen mit dieser Vereindeutigung von Freund und Feind, von Fortschritt und Reaktion verloren. Gleichzeitig war der Bezug auf ein kulturelles Erbe, das es zu verteidigen galt, ein Denkmuster, das in einem breiten politischen Spektrum auch außerhalb der linken Diskurse anschlussfähig war. Die verschiedenen, in der Bündnispolitik miteinander verflochtenen Antifaschismen lassen sich so als Sammlung von kulturellen Praxen, politischen Positionen und Identitäten begreifen, die kulturhistorisch lesbar gemacht werden können (Fitch u. a., 2020, S. 2-3).

Die Pluralität des Antifaschismus in der Zwischenkriegszeit, seine breite und oft disparate Organisationsgrundlage sowie die Erschließung seiner Ideenwelt durch individuelle historische Akteur/innen widerlegen die These einer stringenten kommunistischen Kontrolle dieser Bewegung. Neuere Studien zur Geschichte der Komintern dokumentieren mittlerweile die Ambivalenzen in jener Struktur, die das Politbüro in Moskau mit dieser transnationalen Bewegung vielleicht am direktesten verband (Drachewych, 2019, S. 2-3; Studer \& Dullin, 2018, S. 76-77). Die Unterstellung einer umfassenden Steuerung durch den stalinistischen Machtapparat trägt daher wenig zum historischen Verständnis bei. „The pure and simple assimilation of antifascism to Communism is a retrospective projection of anti-Communist history rather than a judgement formulated on the basis of a contextual analysis", argumentiert etwa Traverso (2016b, S. 261). Wirkung auf den antifaschistischen Organisationskosmos entfaltete die stalinistische Politik insbesondere in der Diskussion ihrer Verbrechen. Die Positionierung zur "Sozialfaschismus-These“, zur auf dem VII. Weltkongress der Komintern in Moskau 1935 etablierten Faschismus-Definition, zu den Schauprozessen und politischer Verfolgung innerhalb der eigenen Reihen begleiteten die antifaschistische Bündnispolitik von Anfang an und führten zu einer Vielzahl von internen Konflikten und Brüchen. 


\section{Dis/Kontinuitäten}

In der Aneignung der politischen Praxis und der Zusammenarbeit mit verschiedenen Bundesgenoss/innen bestanden auch für Antifaschist/innen mit kommunistischen Parteibuch Spielräume, insbesondere, wenn sie sich zwischen mehreren politischen Kontexten, Sprachgemeinschaften und nationalen Zugehörigkeiten bewegten. Nicolaas Rost gehörte zu diesen Übersetzerfiguren, die transnationale Verbindungen aufrechterhielten und ermöglichten. Er selbst konnte sich nie vollständig einer Parteidisziplin unterwerfen. In Belgien arbeitete er für die sozialdemokratische Zeitung "Vooruit" statt für das Organ der Kommunistischen Partei von Belgien. Er beteiligte sich an Kampagnen zur Diskreditierung parteipolitischer Gegner/innen in den Niederlanden, aber auch an Solidaritätskampagnen für kommunistische Abweichler. ${ }^{14}$ In seinen kulturpolitischen Beiträgen porträtierte er russische Sozialrevolutionäre, Pariser Bohémiens und zaristische Polizeiagenten - sehr zum Missfallen seiner Parteifreunde (Rost, 1931). Und im Spanischen Bürgerkrieg, den er als Journalist begleitete, pflegte er freundschaftliche Beziehungen zu Kommunisten wie Trotzkisten gleichermaßen (Rost, n.D., Bl. 11). Bestimmt wurde sein Handeln von einem politischen Pragmatismus und der existenziellen Bedrohung durch den Nationalsozialismus. Um dem zu begegnen, ordnete er politische Loyalitäten der Chance auf einen gemeinsamen Widerstand unter. Solche Ambivalenzen der antifaschistischen Weltaneignung retrospektiv mit einer falschen Eindeutigkeit zu versehen, verstellt den Blick auf dieses historische Problemfeld. Insbesondere das lebensgeschichtliche Einschreiben in antifaschistische Organisationsideen und Diskurse kann nur verstanden werden, wenn man von diesen Verkürzungen abrückt.

Gerade diese individuelle antifaschistische Praxis war es auch, die nach dem Zerbrechen vieler Organisationsformen in Krieg und Exil weiterentwickelt werden sollte. Strukturell geschwächt durch die politische Annährung zwischen der Sowjetunion und dem nationalsozialistischen Deutschland sowie durch die Verfolgung gerade der kommunistischen Antifaschist/innen bleib nach 1939 kaum mehr eine gemeinsame organisatorische Basis. Spätestens mit dem Beginn der deutschen Besatzungsherrschaft in Europa ließen sich die Netzwerke der Zwischenkriegszeit nicht mehr aufrechterhalten. Für viele Antifaschist/innen blieb im Exil nur die Option, unter widrigen Umständen die einstige Bündnispolitik in der Fremde fortzuführen (Acle-Kreysing, 2016, S. 670-673; Palmier, 2006, S. 455-456).

Wer unter dem deutschen Regime seine Arbeit im Untergrund fortsetzen wollte, ging ein enormes Risiko ein und wurde in vielen Fällen deportiert oder ermordet. Unter der sich im Verlauf des Zweiten Weltkrieges radikalisierenden Besatzungsherrschaft gelang es nur wenigen, ihre politische Arbeit unter dem Druck des nationalsozialistischen Verfolgungsapparates fortzusetzen. Die Aktivität im Widerstand, ihre Effektivität und Reich-

14 So zum Beispiel gegen Jef Last (Rost, 1938) und für Max Hoelz (Rost, 1951, Bl. 1).

SLH $10 / 2021$ | p. 9 of 18 


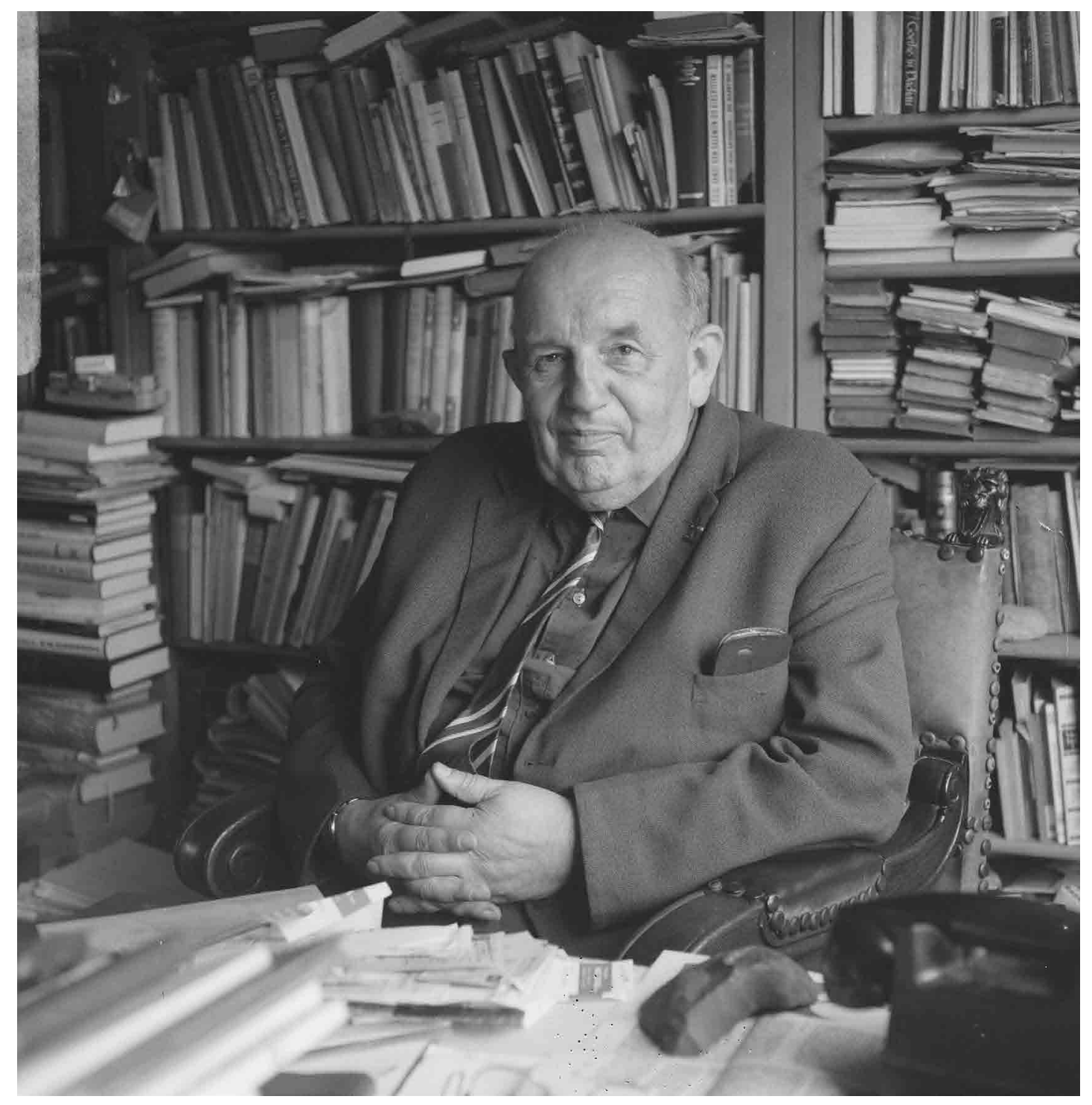

Nicolaas Rost am 21. Juni 1966 in seinem Studierzimmer in Amsterdam zu seinem 70. Geburtstag (Foto: Ron Kroon; Nationaal Archief Den Haag, Fotocollectie Anefo / 919-2704 (CCO 1.0))

weite wurde in den Diskursen der Nachkriegszeit oft überhöht und simplifiziert (Lagrou, 2003, S. 531). Auch die Antifaschist/innen selbst passten ihre Selbsterzählungen nachträglichen diesen Legitimationserzählungen an. Während des Krieges selbst sahen sich viele mit dem Scheitern ihres eigenen Engagements konfrontiert, dessen fehlende Konsequenz die befürchtete Katastrophe nicht hatte verhindern können. Gleichzeitig eröffneten sich neue Perspektiven auf die nationalsozialistischen Verbrechen. Vor Kriegsausbruch war Antisemitismus, etwa in der von Rost beworbenen Quellensammlung Der gelbe Fleck, zwar ein Thema gewesen..$^{15}$ Aber erst mit dem Beginn der rassistischen Vernichtungspolitik im besetzten Osteuropa begannen einzelne Antifaschist/innen, an

15 Zur Interpretation der antisemitischen Verfolgung in diesem Band siehe Dafinger (2016, S. 224). 
der grundlegenden Annahme zu zweifeln, dass der Nationalsozialismus sich zuallererst gegen seine erklärten politischen Gegner/innen richtete (Barck, 2019, S. 231; Koch, 2020, S. 228-231). Insbesondere jüdische Antifaschist/innen und die Überlebenden der Konzentrationslager erlebten diese erfahrungsgeschichtlich begründete Erweiterung der eigenen Perspektive.

Nicolaas Rost war während der ersten Kriegsjahre Mitarbeiter in den kollaborierenden belgischen Verlagen Manteau und De Lage Landen. Außerdem war er Mitglied einer deutschsprachigen Widerstandszelle in Brüssel. Die Spannung zwischen diesen beiden Welten trug auch seine Partnerin Edith Blumberg, die als Jüdin mit ihm zusammen nach Belgien geflüchtet war und dort Gefahr lief, nach Auschwitz-Birkenau deportiert zu werden (Schram, 2017, S. 157-161). Nur mit Glück gelang es, sie mit dem Verweis auf die "Mischehe“ von dem Transport in die Vernichtung auszunehmen. Rost wurde dagegen wegen seiner früheren Aktivitäten gegen den Nationalsozialismus 1943 verhaftet und in das Konzentrationslager Dachau verschleppt. Seine Gefangenschaft, in der er viele Weggefährten aus der Zwischenkriegszeit wiedertraf, interpretierte er nachträglich nach den Mustern, die er sich schon in den Jahren der Volksfront angeeignet hatte. In seinem Erlebnisbericht Goethe in Dachau konstruierte er eine antifaschistische Gemeinschaft, die in der Gefangenschaft dem Nationalsozialismus die Errungenschaften der humanistischen Kultur entgegenhielt (Rost, 1948, S. 134-135).

Diese eingeübte politische Erzählung überspannte das Engagement der Zwischenkriegszeit, die nationalsozialistische Verfolgung und die Nachkriegszeit. Jenseits der organisatorischen Fragmentierung des Antifaschismus in Krieg und Exil schuf eine solche Aneignungserzählung eine sinnstiftende Kontinuität. Die überlebenden Antifaschist/ innen, von denen ein Großteil nach der Befreiung Europas am materialen und moralischen Wiederaufbau mitwirkte, rahmten ihre Aktivitäten in ähnlichen Narrativen. Diese Konstruktion einer Traditionslinie, die die Ikonen der Weimar Klassik gegen das Erbe des Nationalsozialismus in Stellung brachte, beschreibt Agocs in seiner Studie für die sowjetische Besatzungszone und die DDR als antifaschistischen Humanismus (Agocs, 2017, S. 3-5).

Solche individuellen und überindividuellen Kontinuitäten stellen auch die herkömmliche Unterscheidung zwischen dem historischen Antifaschismus vor 1945 und dem Antifaschismus nach der Befreiung in Frage (Groppo, 2004, S. 740-743; Rabinbach, 2009, S. 38). Betrachtet man die historischen Träger/innen des Engagements, ihre Erzählungen und Organisationsideen, rückt das Nebeneinander von Kontinuitäten und Diskontinuitäten in den Mittelpunkt. Zumal die militärische Niederlage des Nationalsozialismus und der mit inm kollaborierenden Regime in der historischen Perspektive längst nicht das Ende des Faschismus bedeutete. Mit den faschistischen Ideologemen, Funktionsträger/innen und Unterstützer/innen musste nicht nur in den NS-Nachfolgestaaten und -gesellschaften in Österreich, Ost- und Westdeutschland ein Umgang gefunden werden. 
In den unmittelbaren Nachkriegsjahren arbeiteten viele Antifaschist/innen an Staatsbildungsprozessen und der Formung nationaler Erinnerungspolitiken in Ost- und Westeuropa mit. Viele befanden sich erstmals in einer Position der Wirkmächtigkeit. Unter dem Schutz der alliierten Besatzungsautoritäten bekamen sie die Chance, in öffentlichen Verwaltungen, als Bürgermeister, im Kulturleben oder im Gerichtssaal ihre Vorstellungen eines gesellschaftlichen Neubeginns einzubringen. In den Nachfolgegesellschaften des nationalsozialistischen Deutschlands, aber auch in den ehemals besetzten Staaten, stießen sie damit allerdings schon früh an die Grenzen, die Reintegration von Täter/innen, Kolaborateur/innen und der Aufbau neuer staatlicher Strukturen setzten. ${ }^{16}$ Für einige führte der Weg durch den Kalten Krieg zurück in die Dissidenz gegenüber den kommunistischen Parteien, die in den verschiedenen Volksdemokratien den Antifaschismus als funktionale Rechtfertigungsideologie nutzten (Oberle, 2012, S. 136). In den individuellen Erfahrungsräumen überdauerte aber auch die alte Pluralität des Antifaschismus, die sich in den 1960er Jahren mit neuen Themen und politischen Bewegungen verband (Vergnon, 2009, S. 163). Ebenso wenig wie sich Antifaschismus also auf einen parteipolitischen „Grundkonsens“ reduzieren lässt, geht er in einer monolithischen kommunistischen Meistererzählung auf. ${ }^{17}$

Nicolaas Rost war mit Goethe in Dachau in der Nachkriegszeit ein bescheidener literarischer Erfolg gelungen. Im ostdeutschen Verlag Volk und Welt erschien das Buch schon 1948 in einer Übersetzung seiner Frau Edith (Ceuppens, 2020, S. 166). Als vermeintliches Zeugnis des humanistischen deutschen Geistes im Angesicht der nationalsozialistischen Verbrechen war das Buch Teil des Zeremoniells im Goethejahr 1949, das mit der Gründung der beiden deutschen Staaten zusammenfiel. Rosts eigene Glaubwürdigkeit als Antifaschist und Überlebender stützte den zentralen Mythos der jungen DDR, die „antifaschistisch-demokratische Umwälzung“ mit einer idealisierten deutschen Kulturgeschichte verbinden zu können (Nothnagle, 1999, S. 42). In gleicher Weise brachte Rost seine Netzwerke aus der Vorkriegszeit in der DDR ein und engagierte sich in der Zeitschrift Blick nach Polen für eine Verständigung mit dem Nachbarstaat. Diese antifaschistische Kulturpolitik sparte die deutschen Verbrechen in der Regel nicht aus, maß positiven Beispielen in der Kulturtradition jedoch eine viel größere Bedeutung bei. ${ }^{18}$

Die von Rost vertretene, oft eigenwillige Interpretation des Antifaschismus wurde inm im Zuge der „volksdemokratischen Homogenisierung“ (Gerber, 2016, S. 211) in den sozialistischen Staaten zum Verhängnis. Im Zuge der Parteiausschlussverfahren und Vorbereitungen zu den antisemitischen Schauprozessen gegen Emigrant/innen und jüdische Kommunist/innen wurde er unter fadenscheinigen Vorwürfen 1951 aus der DDR

16 Dieser Bezug der begrenzten Wirksamkeit trifft am stärksten auf jüdische Remigranten zu, wie Van Laak (2018, S. 165-180) am Beispiel der Sowjetischen Besatzungszone und der DDR beschreibt.

17 Auf eine solche monolithische Erzählung verkürzt auch Grunenberg (1993, S. 138) die Geschichte des Antifaschismus.

18 Siehe etwa die von Rost (1950) konstruierte Verbindung zwischen Goethe und Mickiewicz. 
ausgewiesen. Es sollte fünf Jahre dauern, bis er seine hartnäckigen Rehabilitationsversuche aufgab und den kommunistischen Parteien 1956 den Rücken kehrte. Sein Selbstbild als kommunistischer Antifaschist, der über die politischen Lager hinweg arbeitete, gab er indes nicht auf. ${ }^{19}$ Auch nach seiner öffentlichen Kritik an der sowjetischen Intervention in Ungarn hielt er in Amsterdam Verbindungen zu den kommunistischen Parteien Osteuropas aufrecht.

Der Bruch mit der Disziplin der kommunistischen Parteien ermöglichte es Rost zugleich, sein Verständnis des Antifaschismus um neue historische Bezüge zu erweitern. Für inn bedeutete das, in die Erzählungen des politischen Widerstands und der kulturellen Selbstbehauptung auch jene Opfergruppen miteinzubeziehen, die in den 1950er in Forschung und Öffentlichkeit kaum Beachtung fanden. ${ }^{20}$ Rosts frühe Dokumentation und Interpretation der nationalsozialistischen Verbrechen galt zunächst den jüdischen Gemeinden in den Niederlanden, etwa in seiner Geburtsstadt Groningen. ${ }^{21}$ Zudem unterstützte er Anfang der 1960er Jahre die Bürgerrechtsbewegung der Rom/nja in Frankreich bei ihrem Kampf für Minderheitenrechte und den Zugang zu Wiedergutmachungsleistungen aus Westdeutschland (Sierra-Alonso, 2019, S. 280). Maßgeblich in dieser Erweiterung der antifaschistischen Gemeinschaftskonzeption beeinflusst hatte Rost dabei die israelische Historikerin Miriam Novitch, die er 1951 erstmals in Amsterdam getroffen hatte. ${ }^{22}$ Als jüdische Überlebende hatte Novitch seit 1945 Zeugnisse der Shoah gesammelt und sich dabei insbesondere auf den jüdischen Widerstand in den Vernichtungslagern der „Aktion Rheinhardt“ spezialisiert. ${ }^{23}$ Rost und Novitch gehörten zu den frühen Besucher/innen der ehemaligen Vernichtungsstätten in Polen. Beide versuchten, gerade in Westeuropa Aufmerksamkeit für die Massenverbrechen an den Juden/Jüdinnen herzustellen. Ihr gemeinsames Bemühen verband Rost und Novitch bald freundschaftlich. Der niederländische Antifaschist übernahm insbesondere ihr Motiv, den jüdischen Widerstand gleichberechtigt neben den Protagonist/innen des politisch oder national gerahmten Widerstands darzustellen (Novitch, 1961, S. 2).

Insbesondere in den Netzwerken und Organisationen der Überlebenden des Nationalsozialismus, die wesentlich von den ehemaligen „politischen“ Gefangenen bestimmt wurden (Doerry u. a., 2014, S. 11), versuchte Rost, eine solche erweiterte antifaschistische Geschichtserzählung zu etablieren. Das betraf auch das Comité international de

19 „[I]ch habe mich nicht geändert - politisch und moralisch nicht und werde mich nicht ändern“, schrieb er nach seinen Parteiverfahren an seine Freundin, die Schriftstellerin Anna Seghers (Rost, 1952).

20 Siehe dazu Fritz u. a. (2016, S. 17). Rosts Aktivitäten relativieren auch die dort getroffene Feststellung, dass die frühen Aufarbeitungsprozesse in den 1950er Jahren aufgehört hätten.

21 Siehe insbesondere das Werk De vrienden van m'n vader (übersetzt: Die Freunde meines Vaters) (Rost, 1956).

22 Miriam Novitch, geboren 1908 bei Grodno, gestorben 1990 im Kibbuz Lohamei HaGeta’ot in Israel, gehört zu den in der Forschung kaum beachteten jüdischen Historikerinnen. Im Frankreich der Volksfront-Ära durchlief sie eine ähnliche Politisierung wie Rost. Eine Basis für ihre umfangreichen Dokumentationsbemühungen fand sie im Museum des Lohamei HaGeta'ot, der Kibbuz der Ghettokämpfer in Israel. Von dort aus unternahm Novitch lange Reisen zu den Orten der Vernichtungsstätten der Shoah. Zur Biografie siehe Geva (2015).

23 Siehe beispielsweise Novitch (1946). Das Buch publizierte die Historikerin damals unter dem Pseudonym „Miriam Bath-Ami“. 
Dachau, in dem Rost an der Etablierung einer Gedenkstätte am Ort des ehemaligen Konzentrationslagers mitarbeitete (Marcuse, 2014, S. 171-172). Ebenso wichtig war diese Perspektive für Rost in der medialen Skandalisierung der Weiterbeschäftigung und Wiedereinstellung von nationalsozialistischen Tätern im westdeutschen Staatsdienst. Solche Kontinuitäten wurden von den Antifaschist/innen der Nachkriegszeit als „Renazifizierung“ beschrieben und damit in einen übergeordneten Faschisierungsprozess eingeordnet, dem man mit dem eigenen Engagement begegnen müsse (März, 2016, S. 42, 284). Aus Rosts Perspektive war die Erweiterung des angeeigneten antifaschistischen Horizonts nur konsequent, weil sie mit dem Blick auf die Opfer des Nationalsozialismus das Fortbestehen faschistischer Tendenzen vor allem in Westdeutschland offenlegte.

\section{Fazit}

Eine erfahrungsgeschichtliche Perspektive auf den Antifaschismus ist in all ihren Details schwer reproduzierbar, bietet aber einen Einblick in Pluralität, Entwicklungslinien und Diskontinuitäten. Es ist ein kategorialer Fehler, die Anschlussfähigkeit zu anderen sozialen und politischen Bewegungen allein als Beliebigkeit oder als Summe von Instrumentalisierungen zu begreifen (Bray, 2017, S. 197-199). Zur kritischen Historisierung des Antifaschismus - besser: der Antifaschismen - gehört ebenso die Reflexion seiner Beziehung zur Gegenwart (Dimitrakaki \& Weeks, 2019, S. 288). Solange die Nachwirkungen des historischen Faschismus, die positiven Bezugnahmen auf seine Ideen weiter bestehen, solange wird Antifaschismus ein notwendiger Begriff bleiben - sowohl in der Wissenschaft als auch im gesellschaftlichen Diskurs. Gerade die gegenwärtigen Dynamiken der Faschisierung in vielen Gesellschaften sind ohne ein Bewusstsein für diesen historischen Zusammenhang nicht zu verstehen. Letztendlich ist auch der wissenschaftliche oder intellektuelle Anti-Antifaschismus Teil jener gesellschaftlichen Verhältnisse, die nach der historischen Evidenz von Faschismus, Nationalsozialismus, Shoah und Weltkrieg eine „Neue Rechte“ in Europa hervorgebracht haben. Hinter den Aufrufen zur unpolitischen Distanz gegenüber dem Antifaschismus oder dem Beschwören eines nie vorhandenen „Grundkonsens“ verbirgt sich so auch der Unwille, den eigenen Standpunkt im langen Nachleben des Faschismus bestimmen zu wollen. ${ }^{24}$

24 Ich danke den beiden anonymen Reviewer/innen und Katrin Stoll für die anregenden und hilfreichen Kommentare zum Text. 


\section{Literaturverzeichnis}

Acle-Kreysing, A. (2016). Shattered dreams of anti-fascist unity: German speaking exiles in Mexico, Argentina and Bolivia, 1937-1945. Contemporary European History, 25(4), 667-686. https://doi.org/10.1017/ S0960777316000436

Agocs, A. (2017). Antifascist humanism and the politics of cultural renewal in Germany. Cambridge University Press. https://doi.org/10.1017/9781316084106

Albert, G. J. (2017). Das Charisma der Weltrevolution: Revolutionärer Internationalismus in der frühen Sowjetgesellschaft 1917-1927. Böhlau Verlag. https://doi.org/10.7788/9783412508579

Barck, S. (2019). Das verhinderte Schwarzbuch: Wassili Grossman und Ilja Ehrenburg und der Genozid an den sowjetischen Juden. In H. Peitsch (Ed.), Nachkriegsliteratur als öffentliche Erinnerung: Deutsche Vergangenheit im europäischen Kontext (pp. 231-245). De Gruyter. https://doi.org/10.1515/9783050093932-011

Barrett, N. (1999). The anti-fascist movement in south-east Lancashire. In T. Kirk \& A. McElligoot (Eds.), Opposing fascism: Community, authority and resistance in Europe (pp. 48-62). Cambridge University Press. https://doi.org/10.1017/CB09780511497070.005

Bayerlein, B. H. (1996). Einheitsfront- und Volksfrontmythos als Ursprungslegenden des Antifaschismus. In C. Keller (Ed.), „Der Verräter, Stalin, bist Du!“ Vom Ende der linken Solidarität: Komintern und kommunistische Parteien im Zweiten Weltkrieg 1939-1941 (pp. 103-122). Aufbau-Taschenbuch-Verlag.

Bayerlein, B. H., Braskén, K., \& Weiss, H. (2017). Transnational and global perspectives on international communist solidarity organisations. In H. Weiss (Ed.), International communism and transnational solidarity: Radical networks, mass movements and global politics, 1919-1939 (pp. 1-27). Brill. https://doi. org/10.1163/9789004324824_002

Behrends, J. C. (2020, June 3). Warum ich kein Antifa bin - eine historische Einordnung. Salonkolumnisten. https://www.salonkolumnisten.com/warum-ich-kein-antifa-bin-eine-historische-einordnung/

Braskén, K. (2015). The international workers' relief, communism, and transnational solidarity: Willi Münzenberg in Weimar Germany. Palgrave Macmillan. https://doi.org/10.1057/9781137546869

Braskén, K. (2016). Making anti-fascism transnational: The origins of communist and socialist articulations of resistance in Europe, 1923-1924. Contemporary European History, 25(4), 573-596. https://doi. org/10.1017/S0960777316000424

Braskén, K., Copsey, N., \& Featherstone, D. J. (Eds.). (2020). Anti-fascism in a global perspective: Transnational networks, exile communities, and radical internationalism. Routledge. https://doi.org/10.4324/9780429058356

Bray, M. (2017). Antifa: The anti-fascist handbook. Melville House.

Bray, M., Namakkal, J., Riccò, G., \& Roubinek, E. (2020). Editors' introduction. Radical History Review, 2020(138), 1-9. https://doi.org/10.1215/01636545-8359223

Ceuppens, J. (2020). Rückübersetzung: The fates of Nico Rost's diary Goethe in Dachau. Dutch Crossing, 44(2), 165-180. https://doi.org/10.1080/03096564.2020.1747007

Copsey, N. (2010). Preface: Towards a new anti-fascist "minimum"? In N. Copsey \& A. Olechnowicz (Eds.), Varieties of anti-fascism: Britain in the inter-war period (pp. xiv-xxi). Palgrave Macmillan.

Dafinger, S. (2016). „viel schauerliches und viel groteskes“: Lion Feuchtwangers Deutung der nationalsozialistischen Judenverfolgung. In B. Bannasch, H. Schreckenberger, \& A. E. Steinweis (Eds.), Exil und Shoah (pp. 205-225). edition text + kritik.

De Felice, R. (1995). Rosso e nero (3rd ed.). Baldini \& Castoldi.

Dimitrakaki, A., \& Weeks, H. (2019). Anti-fascism/Art/Theory: An introduction to what hurts us. Third Text, 33(3), 271-292. https://doi.org/10.1080/09528822.2019.1663679

Doerry, J., Kubetzky, T., \& Seybold, K. (2014). Einleitung. In J. Doerry, T. Kubetzky, \& K. Seybold (Eds.), Das soziale Gedächtnis und die Gemeinschaften der Überlebenden: Bergen-Belsen in vergleichender Perspektive (pp. 9-32). Wallstein. 
Drachewych, 0. (2019). The Communist Transnational? Transnational studies and the history of the Comintern. History Compass, 17(2), 1-12. https://doi.org/10.1111/hic3.12521

Esken, S., \& Walter-Borjans, N. (2020, February 6). Kein Fußbreit dem Faschismus! Sozialdemokratische Partei Deutschlands (SPD). https://www.spd.de/aktuelles/detail/news/kein-fussbreit-dem-faschismus/06/02/2020/

Featherstone, D. (2018). Anti-fascism, anti-colonialism and the making of solidarities. In B. Bayerlein, K. Braskén, \& U. Sonnenberg (Eds.), Globale Räume für radikale transnationale Solidarität: Beiträge zum Ersten Internationalen Willi-Münzenberg-Kongress 2015 in Berlin (pp. 39-65). Rosa-Luxemburg-Stiftung; Münzenbergforum Berlin.

Fitch, M., Ortiz, M., \& Underwood, N. (2020). Editorial introduction: The global cultures of antifascism, 1921-2020. Fascism, 9(1-2), 1-7. https://doi.org/10.1163/22116257-20201199

Fritz, R., Kovács, É., \& Rásky, B. (2016). Der NS-Massenmord an den Juden: Perspektiven und Fragen der frühen Aufarbeitung. In R. Fritz, É. Kovács, \& B. Rásky (Eds.), Als der Holocaust noch keinen Namen hatte: Zur frühen Aufarbeitung des NS-Massenmordes an den Juden / Before the Holocaust had its name: Early confrontations of the Nazi mass murder of the Jews (pp. 7-19). New Academic Press.

Furet, F. (1995). Le passé d'une illusion: Esssai sur l'idée communiste au XXe siècle. Laffont.

García, H. (2016). Was there an antifascist culture in Spain during the 1930s? In H. García, Y. Rodrigo, X. Tabet, \& C. Clímaco (Eds.), Rethinking antifascism: History, memory and politics, 1922 to the present (pp. 92-113). Berghahn. https://doi.org/10.2307/j.ctvpj7j84.9

Gerber, J. (2016). Ein Prozess in Prag: Das Volk gegen Rudolf Slánský und Genossen. Vandenhoeck \& Ruprecht. https://doi.org/10.13109/9783666370472

Geva, S. (2015). "To collect the tears of the Jewish people": The story of Miriam Novitch. Holocaust Studies, 21(1-2), 73-92. https://doi.org/10.1080/17504902.2015.1062276

Groppo, B. (2004). Fascismes, antifascismes et communismes. In M. Dreyfus, B. Groppo, C. S. Ingerflom, R. Lew, C. Pennetier, B. Pudal, \& S. Wolikow (Eds.), Le siècle des communismes (2nd ed., pp. 739-758). Editions de l'Atelier.

Großmann, J. (2016). Die „Grundtorheit unserer Epoche“? Neue Forschungen und Zugänge zur Geschichte des Antikommunismus. Archiv für Sozialgeschichte, 56, 549-590.

Grunenberg, A. (1993). Antifaschismus: Ein deutscher Mythos. Rowohlt-Taschenbuch-Verlag.

Hobsbawm, E. J. (1985). Fifty years of People's Fronts. In J. Fyrth (Ed.), Britain, fascism and the Popular Front (pp. 235-250). Lawrence and Wishart.

Jesse, E. (2021, April 21). Die Antifaschismus-Falle: Antifaschismus ist in aller Munde: Weshalb Faschismus in Deutschland nicht als Warnformel taugt. Neue Zürcher Zeitung.

Karimäki, J. (2019). Finnish liberals and antifascism, 1922-1932. In K. Braskén, N. Copsey, \& J. A. Lundin (Eds.), Anti-fascism in the Nordic Countries: New perspectives, comparisons and transnational connections (pp. 39-54). Routledge. https://doi.org/10.4324/9781315171210-3

Koch, A. (2020). Exile dreams: Antifascist Jews, antisemitism and the "other Germany". Fascism, 9(1-2), 221-243. https://doi.org/10.1163/22116257-20201171

Kusicke, S. (2020, February 14). „Zweierlei Maß verbietet sich“: Muss die CDU den gleichen Abstand nach rechts und links außen wahren? Ein Gespräch mit dem Extremismusforscher Eckhard Jesse über das Dilemma in Thüringen. Frankfurter Allgemeine Zeitung.

Lagrou,P. (2003). The politics of memory: Resistance as a collective myth in post-war France, Belgium and the Netherlands, 1945-1965. European Review, 11(4), 527-549. https://doi.org/10.1017/S1062798703000474

Langkau-Alex,U. (2004). Deutsche Volksfront 1932-1939: Vol. 1. Vorgeschichte und Gründung des Ausschusses zur Vorbereitung einer deutschen Volksfront (2nd ed.). Akademie Verlag. https://doi.org/10.1515/9783050047492

Leggewie, C. (2020, February 7). Wer sich mit der AfD einlässt, verrät die Demokratie. Frankfurter Rundschau. https://www.fr.de/politik/thueringen-sich-einlaesst-verraet-demokratie-13526230.html

Marcuse, H. (2014). Die Organisationen der Überlebenden von Dachau: Ein Abriss der Entwicklung von Befreiung des Konzentrationslagers bis Anfang der 1970er Jahre. In J. Doerry, T. Kubetzky, \& K. Seybold 
(Eds.), Das soziale Gedächtnis und die Gemeinschaften der Überlebenden: Bergen-Belsen in vergleichender Perspektive (pp. 159-174). Wallstein.

März, J. (2016). Zwischen Politik und Interessenvertretung: Die Verbände der politischen Opfer des Nationalsozialismus in der Bundesrepublik Deutschland von 1947 bis 1990 [Doctoral Dissertation]. Universität zu Köln.

Nothnagle, A. L. (1999). Building the East German myth: Historical mythology and youth propaganda in the German Democratic Republic, 1945-1989. University of Michigan Press. https://doi.org/10.3998/mpub.15410

Novitch, M. (1946). Le droit d'avoir: Témoignages sur les massacres des Juifs en Pologne. L. Rodstein.

Novitch, M. (1961). The Jewish Resistance and the allies. In Museo della Szienza e della Tecnica (Ed.), Secondo congresso internationale di storia della resistenza / Deuxieme conference internationale d'histoire de la resistance / Second International Conference on the History of the Resistance. Milano, 26/29 Marzo 1961 (Vol. 2, pp. 1-67). Selbstverlag des Museums.

Oberle, C. M. (2012). Reconfiguring postwar antifascism: Reflections on the history of ideology. New German Critique, 117, 135-153. https://doi.org/10.1215/0094033X-1677300

Olink, H. (1997). Nico Rost: De man die van Duitsland hield: Een biografische schets. Nijgh \& Van Ditmar.

Palmier, J.-M. (2006). Weimar in exile: The antifascist emigration in Europe and America. Verso.

Petersson, F. (2013). “We are neither visionaries nor utopian dreamers": Willi Münzenberg, the League against Imperialism, and the Comintern, 1925-1933 [Doctoral Dissertation, Åbo Akademi University]. Åbo Akademis förlag. https://www.doria.fi/bitstream/handle/10024/90023/petersson_fredrik.pdf

Rabinbach, A. (2009). Begriffe aus dem Kalten Krieg: Totalitarismus, Genozid und Geschichtsschreibung. Wallstein.

Racine-Furlaud, N. (1977). Le Comité de vigilance des intellectuels antifascistes (1934-1939): Antifascisme et pacifisme. Le Mouvement Social, 101, 87-113. https://doi.org/10.2307/3777881

Raunig, G. (2017). Kunst und Revolution: Künstlerischer Aktivismus im langen 20. Jahrhundert (2nd ed.). transversal texts.

Rost, N. (n.d.). Mein Freund Carl Einstein [Unpublished Manuscript]. Akademie der Künste (Carl-Einstein-Archiv / 506), Berlin.

Rost, N. (1919, November 22). Indrukken uit Berlijn. Provinciale Geldersche en Nijmeegsche courant.

Rost, N. (1926). Brief aus Holland. Der Querschnitt, 6(8), 583-586.

Rost, N. (1931, December 23). Naar aanleiding van een boekbespreking. De Tribune. Revolutionair Volksblad: Orgaan der Communistische Partij in Nederland.

Rost, N. (1933). Links-Richten presenteert U: Een concentratiekamp in het Derde Rijk: (De brouwerij van Oranienburg).

Rost, N. (1938). Het geval Jef Last: Over fascisme en trotzkisme. Pegasus.

Rost, N. (1948). Goethe in Dachau: Literatuur en werkelijkheid. Veen.

Rost, N. (1950). Polen kennt Goethe / Kennt Deutschland Adam Mickiewicz? Blick nach Polen, 1950(10), $13-15$

Rost, N. (1951). Levensbericht. Internationaal Instituut voor Sociale Geschiedenis (ARCH02020, 90), Amsterdam.

Rost, N. (1952, December). Brief an Anna Seghers. Akademie der Künste (Anna-Seghers-Archiv / 1454), Berlin.

Rost, N. (1956). De vrienden van m'n vader: Herinneringen aan de Folkingestraat. Van Gorcum.

Rother, B., Meyer, K., \& Morina, C. (2020, March 25). 156 Jahre Faschismus? Für klare Begriffe in unklaren Zeiten. Sozialdemokratische Partei Deutschlands (SPD). https://geschichtsforum.spd.de/debatten/fuer-klare -begriffe-in-unklaren-zeiten/

Schram, L. (2017). Dossin: L'antichambre d'Auschwitz. Racine. 
Schildt A. (2017). Antikommunismus von Hitler zu Adenauer. In N. Frei \& D. Rigoll (Eds.), Der Antikommunismus in seiner Epoche: Weltanschauung und Politik in Deutschland, Europa und den USA (pp. 186-203). Wallstein.

Sierra-Alonso, M. (2019). Creating Romanestan: A place to be a Gypsy in post-Nazi Europe. European History Quarterly, 49(2), 272-292. https://doi.org/10.1177/0265691419836909

Späth, J. (2019, February 4). Antifaschismus: Begriff, Geschichte und Forschungsfeld in westeuropäischer Perspektive [Version 1.0]. Docupedia Zeitgeschichte. https://doi.org/10.14765/zzf.dok-1323

Studer, B., \& Dullin, S. (2018). Communism + transnational: The rediscovered equation of internationalism in the Comintern years. Twentieth Century Communism, 14, 66-95. https://doi.org/10.3898/175864318823243726

Traverso, E. (2016a). Antifascism between collective memory and historical revisions. In H. García, Y. Rodrigo, X. Tabet, \& C. Clímaco (Eds.), Rethinking antifascism: History, memory and politics, 1922 to the present (pp. 321-338). Berghahn. https://doi.org/10.2307/j.ctvpj7j84.21

Traverso, E. (2016b). Fire and blood: The European Civil War 1914-1945. Verso.

Van Laak, J. (2018). Eine Erfahrungsgeschichte der Rückkehr: Jüdische Emigranten-Ehepaare über ihre ersten Jahre in der SBZ/DDR. Jahrbuch des Dubnow-Instituts / Dubnow Institute Yearbook, 17, 155-182.

Vergnon, G. (2009). L'antifascisme en France: De Mussolini à Le Pen. Presses Universitaires de Rennes. https:// doi.org/10.14375/NP.9782753509511

Wegewitz, M. (2021). Kultivierter Antifaschismus: Nicolaas Rost und der lange Kampf gegen den Nationalsozialismus, 1919-1967 [Unpublished Doctoral Dissertation]. Friedrich-Schiller-Universität Jena.

Wildt, M. (2019). Die Ungleichheit des Volkes: „Volksgemeinschaft“ in der politischen Kommunikation der Weimarer Republik. In M. Wildt, Die Ambivalenz des Volkes: Der Nationalsozialismus als Gesellschaftsgeschichte (pp. 47-65). Suhrkamp.

\section{Categorical Mistakes: Antifascism in Public Discourse and Transnational Biography}

Abstract: This essay examines some of the recent tendencies to delegitimize antifascism both as a political phenomenon and as a topic of historical research. In German academia and beyond, this "anti-antifascist" current operates according to notions of communist contamination. To counter these narratives, I propose to look at the plurality of antifascism, its various continuities and the personal experiences of its historical agents. In particular, I make my case exploring the transnational biography of the Dutch journalist Nicolaas Rost.

Keywords: antifascism; biography; communism; historiography; transnational history

Citation: Wegewitz, M. (2021). Kategorienfehler: Antifaschismus im öffentlichen Diskurs und transnationaler Biografik. Studia Litteraria et Historica, 2021(10), Article 2569. https://doi. org/10.11649/slh.2569

This is an Open Access article distributed under the terms of the Creative Commons Attribution 3.0 PL License, which permits redistribution, commercial and non-commercial, provided that the article is properly cited. http://www.creativecommons.org/licenses/by/3.0/pl (c) The Author(s) 2021

Publisher: Institute of Slavic Studies, Polish Academy of Sciences, Warsaw, Poland Author: Markus Wegewitz, Buchenwald and Mittelbau-Dora Memorials Foundation, Germany ORCID: https://orcid.org/0000-0002-5183-9899

Correspondence: mwegewitz@buchenwald.de

The preparation of this work was self-financed by the author.

Competing interests: The author has declared he has no competing interests.

Publication history: Received: 2021-05-04; Accepted: 2021-05-26; Published: 2021-12-31 